\title{
Attending to horizontal, diagonal, and vertical positions in space
}

\author{
YEHOSHUA TSAL \\ Tel Aviv University, Tel Aviv, Israel
}

\begin{abstract}
Vocal reaction times were measured for a target letter presented in one of eight possible locations surrounding fixation. The letter was preceded by a single dot marking its expected location (focused attention condition); by a pair of diametrically opposite dots, one of which marked the expected location of the target (divided attention condition); or by eight dots occupying all eight positions in which the target may appear (distributed attention condition). Under all three conditions, targets occupying horizontal positions were responded to faster than those presented in other positions. Moreover, under the focused attention condition, diagonal targets were responded to faster than vertical targets. The results suggest an uneven distribution of attention across the visual field.
\end{abstract}

The present study was designed to assess the distribution of attention over visual space. Specifically, the purpose was to investigate whether attention is uniformly allocated in all directions or whether some regions are more effectively attended to than others. Subjects were presented with one of two possible target letters that appeared in one of eight possible locations surrounding fixation. The letter was preceded by a single dot marking its expected location (focused attention condition); by a pair of diametrically opposite dots, one of which marked the expected location of the target (divided attention condition); or by eight dots occupying all possible positions in which the target may appear (distributed attention condition). The presentation of these three conditions enabled separation of the effects into two possible attentional factors. If different attentional effects for different positions reflect a more effective orienting of attention to some regions in space, then the effects should be substantially more pronounced for the focused attention condition, which cued the precise location of the target. However, if these effects reflect differential attentional sensitivity over the visual field, which is independent of the act of directing attention, then similar effects should be obtained under all three conditions.

\section{METHOD}

\section{Subjects}

The subjects were 12 paid M.I.T. undergraduates. All had normal or corrected-to-normal vision.

\section{Stimuli}

The task was to verbally respond as fast as possible to one of two possible letters. Every subject was presented with two pairs of letters: 4 subjects received the pairs DO and QX, 4 received the pairs GQ and

Requests for reprints should be addressed to Yehoshua Tsal, Department of Psychology, Tel Aviv University, Ramat Aviv, 69978, Tel Aviv, Israel.
DK, and 4 received the pairs KX and GO. The purpose was to enable generalization of the results over varied letter types presented in different combinations. A letter was presented in one of eight possible locations corresponding to the two horizontal, two vertical, and four diagonal positions of an imaginary circle. The circle subtended $8^{\circ}$ of visual angle in diameter and was centered on the fixation cross. A letter subtended a visual angle of $.6^{\circ}$ in height and $.3^{\circ}$ in width. The dot indicator subtended a visual angle of about $.3^{\circ}$. The dots were placed inside the imaginary circle, and their nearest position was about $.06^{\circ}$ from the indicated letter. The experiment was programmed on a DEC PDP$11 / 03$ computer, and the stimuli were displayed on a Terak $8510 \mathrm{a}$ monitor.

\section{Procedure}

The subject was seated in front of the Terak screen with his/her head fixed on a chinrest. The fixation cross appeared on the center of the screen at eye level. Five minutes were allowed for dark adaptation. Each trial began with the onset of the fixation cross, which remained on for the entire trial. One second following the onset of the cross, the dot indicator(s) appeared for $100 \mathrm{msec}$. Thirty-three milliseconds after the offset of the dot(s), the target letter appeared for $50 \mathrm{msec}$. The vocal reaction time to identify the letter was measured.

Each subject was run under three conditions. In the focused attention condition, a single dot was presented near the location of the target. In the divided attention condition, a pair of diametrically opposite dots was presented; each cued the correct target location on half of the trials. In the distributed attention condition, eight dots were presented, occupying all possible locations of the target. Each condition included three blocks of trials. The first block consisted of 72 trials and was used for practice. It contained the letters $\mathrm{H}$ and $\mathrm{L}$ for targets. Its purpose was to provide extensive practice with the particular attention condition prior to introducing the actual experimental targets. Each of the remaining two blocks included one of the two pairs of letters. Each block consisted of $\mathbf{8 0}$ trials, in which the first $\mathbf{1 6}$ were used for practice. In every block, each of the two letters and each of the eight locations appeared with equal frequency and were randomly intermixed. The order of condition presentation was counterbalanced across subjects.

\section{RESULTS AND DISCUSSION}

The mean error rate was $2.69 \%$ and tended to covary with reaction time across conditions. Reaction times were first computed separately for each of the eight positions. An initial analysis indicated neither a right versus left nor 
an upper versus lower visual field difference. Thus, the data were divided into three groups: the two horizontal positions, the two vertical positions, and the four diagonal positions. Figure 1 shows mean reaction times as a function of position for each of the three conditions. A threeway analysis of variance (condition $\times$ position $\times$ subjects) indicated that all effects were significant $[F(2,22)$ $=15.53, p<.0001$, for condition; $F(2,22)=32.27$, $p<.0001$, for position; and $F(4,44)=2.70, p<.05$, for their interaction]. The main effect for condition replicates the general finding of faster responses to a single stimulus with greater location certainty (e.g., Eriksen \& Hoffman, 1974; Posner, Snyder, \& Davidson, 1980). Further analyses revealed that targets in the horizontal positions were responded to faster than those in the diagonal positions under all three conditions $[F(1,11)=$ $4.90, p<.05$, for the focused attention condition; $F(1,11)=21.34, p<.005$, for the divided attention condition; and $F(1,11)=9.67, p<.01$, for the distributed attention condition]. Targets in the diagonal positions were responded to faster than those in the vertical positions, but only under the focused attention condition $[F(1,11)$ $=11.22, p<.01]$.

As Figure 1 indicates, these position effects were not more pronounced under the focused attention condition. If anything, the difference between reaction times for horizontal targets and those for either diagonal or vertical targets was larger under the divided attention condition. Hence, these effects do not seem to be associated with the effectiveness of orienting attention to different regions in space. In the divided and distributed attention

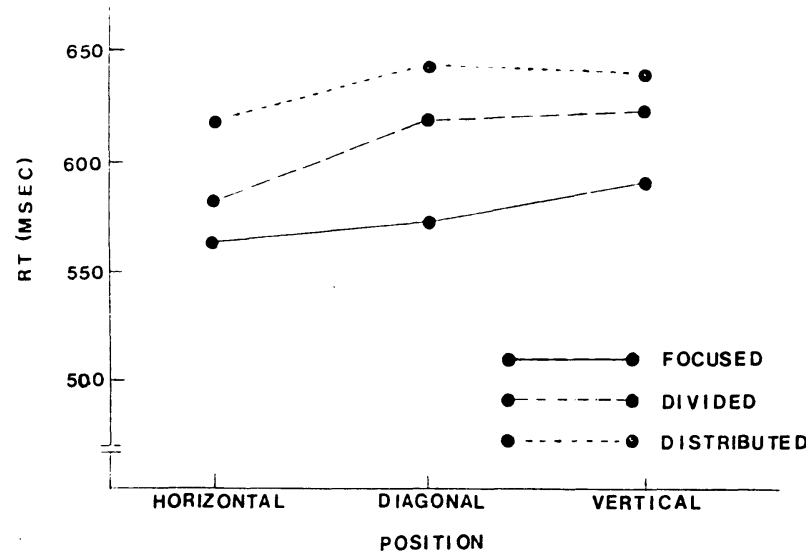

Figure 1. Mean reaction time to vocalize the target as a function of spatial position and attention condition. conditions, attention was at least initially distributed across a large region or over several positions in space; it may have been redirected to the target location upon the target's occurrence. In the focused attention condition, on the other hand, attention could be directed to a specific location upon the onset of the cue and prior to the appearance of the letter. If the position effects obtained in the present study were produced by the mechanism of orienting attention, then these effects should have been stronger under the focused attention condition. Therefore, these effects seem to reveal an uneven distribution of attention that is inherently related to the organization of visual space and is independent of the act of directing attention.

Interestingly, the present pattern of results is similar to that obtained by Skelton and Eriksen (1976), who used an entirely different task. They presented circular arrays of eight letters and instructed subjects to match two of the letters, which were cued peripherally by two bar indicators. When the cued letters were diametrically opposite, matching latencies indicated best performance for the horizontal pairs, second best performance for the diagonal pairs, and worst performance for the vertical pairs. This asymmetry does not seem to be confined to a particular retinal location: in the present study the letters were presented at $4^{\circ}$ from fixation, whereas Skelton and Eriksen presented their letters at $2^{\circ}$ from fixation. Furthermore, Posner (e.g., 1980) reported that the magnitude of attentional costs and benefits remain the same at different peripheral distances along the horizontal axis. Thus, although a more complete study is required to map the sensitivity of the entire attentional field, the above results taken together suggest that the field could be tentatively characterized by uniform sensitivity along axes, with the highest sensitivity along the horizontal axis and gradually declining as the axis moves progressively toward the vertical.

\section{REFERENCES}

Eriksen, C. W., \& Hoffman, J. E. (1974). Selective attention: Noise suppression or signal enhancement? Bulletin of the Psychonomic Society, 4, 587-589.

Posner, M. I. (1980). Orienting of attention. Quarterly Journal of Experimental Psychology, 32, 3-25.

Posner, M. I., Snyder, C. R., \& Davidson, B. J. (1980). Attention and the detection of signals. Journal of Experimental Psychology: General, 109, 160-174.

Skelton, J. M., \& ERIKSEN, C. W. (1976). Spatial characteristics of selective attention in letter matching. Bulletin of the Psychonomic Society, 7, 136-138.

(Manuscript received August 22, 1988.) 